\title{
A noninhibitory mutant of the caveolin-1 scaffolding domain enhances eNOS-derived NO synthesis and vasodilation in mice
}

\author{
Pascal Bernatchez, ${ }^{1}$ Arpeeta Sharma, ${ }^{1}$ Philip M. Bauer, ${ }^{2}$ Ethan Marin, ${ }^{2}$ and William C. Sessa $^{2}$ \\ ${ }^{1} T$ The James Hogg Research Centre, Heart and Lung Institute at St. Paul's Hospital, Department of Anesthesiology, \\ Pharmacology and Therapeutics, University of British Columbia, St. Paul's Hospital, Vancouver, British Columbia, Canada. \\ 2Department of Pharmacology and Vascular Biology and Therapeutics Program, Yale University School of Medicine, New Haven, Connecticut, USA
}

\begin{abstract}
Aberrant regulation of eNOS and associated NO release are directly linked with various vascular diseases. Caveolin-1 (Cav-1), the main coat protein of caveolae, is highly expressed in endothelial cells. Its scaffolding domain serves as an endogenous negative regulator of eNOS function. Structure-function analysis of Cav-1 has shown that phenylalanine 92 (F92) is critical for the inhibitory actions of Cav-1 toward eNOS. Herein, we show that F92A-Cav-1 and a mutant cell-permeable scaffolding domain peptide called Cavnoxin can increase basal NO release in eNOS-expressing cells. Cavnoxin reduced vascular tone ex vivo and lowered blood pressure in normal mice. In contrast, similar experiments performed with eNOS- or Cav-1-deficient mice showed that the vasodilatory effect of Cavnoxin is abolished in the absence of these gene products, which indicates a high level of eNOS/Cav-1 specificity. Mechanistically, biochemical assays indicated that noninhibitory F92A-Cav-1 and Cavnoxin specifically disrupted the inhibitory actions of endogenous Cav-1 toward eNOS and thereby enhanced basal NO release. Collectively, these data raise the possibility of studying the inhibitory influence of Cav-1 on eNOS without interfering with the other actions of endogenous Cav-1. They also suggest a therapeutic application for regulating the eNOS/Cav-1 interaction in diseases characterized by decreased NO release.
\end{abstract}

\section{Introduction}

$\mathrm{NO}$ is well known to actively regulate vascular tone and blood pressure (BP). eNOS is the main source of vascular NO, and aberrant regulation of eNOS activity is linked to a range of vascular diseases. eNOS is a dually acylated protein that targets primarily to the cytoplasmic aspect of the Golgi complex and to plasmalemmal microdomains in ECs called caveolae $(1,2)$. Caveolae organelles are cholesterol and sphingolipid rich, highly abundant in the endothelium and other specialized cells, and are important for various physiological functions (3). When localized to caveolae, the activity of eNOS, and thus the production of NO, under resting conditions is tonically suppressed in a reversible manner via its interaction with the caveolae coat protein caveolin-1 (Cav-1) (4-6). Genetic loss of Cav-1 in mice results in an almost complete loss of the caveolae organelle in vivo, leading to derangements in lipid metabolism, pulmonary hypertension and fibrosis, NO dysfunction, and cardiac abnormalities $(7,8)$, demonstrating the importance of Cav-1 in normal physiology. Although there are many potential mechanisms to explain why the loss of Cav-1 causes these phenotypes, the pulmonary, vascular, and cardiac abnormalities can be rescued by inhibitors of NOS $(9,10)$ or via crossing Cav-1deficient mice to eNOS-deficient mice (11), implying dysregulation of eNOS contributes to aspects of these phenotypes.

Cav-1 is a $25-\mathrm{kDa}$ cholesterol binding protein that forms more than $250 \mathrm{kDa}$ homo-oligomers of greater than 10 monomers, and oligomerization is thought to be important for its function as a scaffold and for organelle assembly $(3,12-14)$. Functional

Authorship note: Pascal Bernatchez and Arpeeta Sharma contributed equally to this work.

Conflict of interest: The authors have declared that no conflict of interest exists. Citation for this article: J Clin Invest. 2011;121(9):3747-3755. doi:10.1172/JCI44778. mapping of the inhibitory interaction of Cav-1 with eNOS using recombinant proteins, yeast 2 -hybrid analysis, and coprecipitation experiments have revealed that a major interacting domain for eNOS on Cav-1 is the putative scaffolding domain of Cav-1 (aa 82-101) $(4,6)$. One model for activation of eNOS bound to Cav-1 is that upon stimulation with calcium-mobilizing agonists, the inhibitory clamp of Cav-1 is relieved via calcium-regulated binding of calmodulin and hsp90 to displace eNOS from Cav-1, thus allowing for efficient NO production (15-17). Evidence supporting the inhibition model includes enhanced NO-dependent vascular function in blood vessels from Cav-1 $\mathrm{KO}$ mice and increased production of $\mathrm{NO}$ in $\mathrm{EC}$ isolated from Cav-1 $\mathrm{KO}$ mice, an effect rescued by reintroduction of Cav-1 $(7,8,18,19)$. In addition, transduction of cells or blood vessels with a cell-permeable version of the scaffolding domain termed Cavtratin reduces $\mathrm{NO}$ release and reduces inflammation in vivo (20-22). Alanine scanning of this scaffolding region demonstrated that the threonine residues 90 and 91 (T90, T91) and in particular phenylalanine 92 (F92), are responsible for eNOS inhibition; this is supported by evidence showing lack of eNOS inhibition by the F92A-Cav-1 mutant in reconstituted cells and a Cavtratin-derived peptide with the T90/91 and F92 substitutions (a peptide called Cavnoxin) using 3 distinct assays in vitro and in vivo (23).

Herein we document that overexpression of the F92A-Cav-1 mutant protein can increase basal NO release from ECs. Similarly, Cavnoxin increases EC NO release, reduces vessel tone ex vivo, and lowers BP in normal mice. The effects of Cavnoxin are lost in vessels from eNOS and Cav-1-deficient mice, indicating that Cavnoxin's effects are highly specific. Mechanistically, we show that the F92A substitution in Cav-1 does not affect its oligomerization state, cellular localization, or ability to interact with eNOS and that both F92A-Cav-1 and Cavnoxin act as 
A

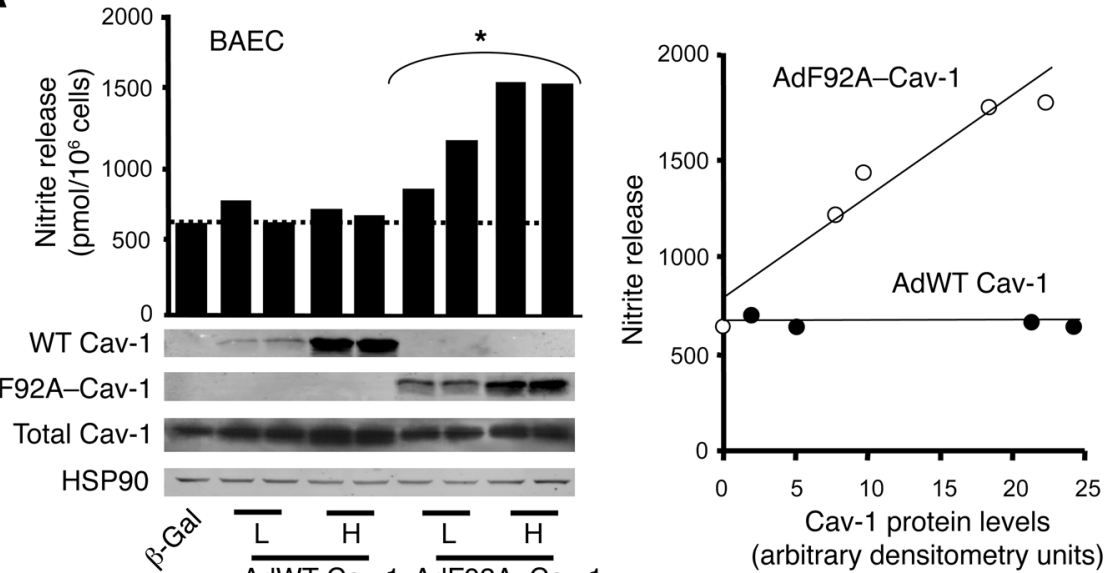

B
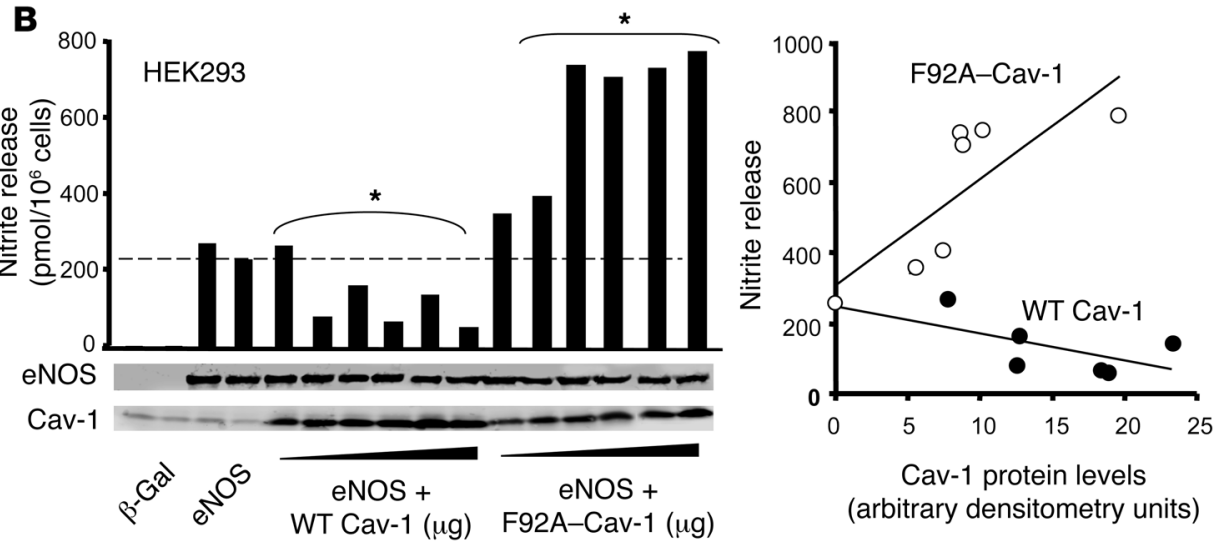

Cav-1 protein levels (arbitrary densitometry units)

Figure 1

F92A-Cav-1 increases NO release. (A) Left panel shows confluent BAECs were infected with low (5 MOI) or high (50 MOI) of purified adenoviruses, and the accumulation of nitrite in medium was quantified after 16 hours by NO-specific chemiluminescence. Expression of WT (myc tagged) and F92A-Cav-1 (HA tagged) was confirmed by Western blotting. Data are individual values of nitrite per $1 \times 10^{6}$ cells and correlate with the Western blotting data below, performed in triplicate. ${ }^{\star} P<0.001$ compared with $\beta$-gal-infected ECs. Right panel shows correlation depicting enhanced nitrite accumulation in cells expressing F92A-Cav-1 versus WT Cav-1 as a function of viral Cav-1 expression (nitrite accumulation per $1 \times 10^{6} \mathrm{cells} / \mathrm{AU}$ of Cav-1 expressed). (B) Left panel shows cotransfection of F92A-Cav-1 cDNA with eNOS cDNA increases nitrite accumulation in HEK293 cells, whereas cotransfection with WT Cav-1 cDNA decreases nitrite accumulation. Cells were transfected with fixed amounts of eNOS cDNA and increasing amount of HA-tagged Cav-1 constructs. Individual nitrite values and correlating Western blot data are shown. ${ }^{\star} P<0.001$ compared with eNOS-expressing cells. Right panel shows correlation depicting increased and decreased nitrite release from cells transfected with F92A or WT Cav-1 cDNAs, respectively. Similar experiments were repeated 2 additional times.

"dominant negative" scaffolding domains, i.e., they relieve eNOS from the inhibitory influence of endogenous Cav-1. In light of the importance of endogenous NO for vascular homeostasis, our data are the first, to our knowledge, to provide direct evidence for augmentation of eNOS NO release by modulating the eNOS/Cav-1 interaction under basal conditions without interfering with basic functions of Cav-1 and suggest that modulating the eNOS/Cav-1 interaction may improve endothelial function in vascular disease.

\section{Results}

F92A mutation of Cav-1 increases NO release. Previously, we have shown that F92A substitution in the Cav-1 cDNA causes loss of eNOS inhibitory activity in reconstituted cells (23). To further test the function of F92A-Cav-1 1 in ECs, we generated adenoviruses encoding WT Cav-1 (myc tagged) and F92A-Cav-1 (HA tagged) for infection into bovine aortic EC (BAEC), which expresses ample levels of endogenous eNOS and Cav-1. Infection of confluent ECs with Ad WT Cav-1 (10 or 50 MOI) did not decrease basal NO release (quantified as nitrite in the medium using a NO-specific chemiluminescence analyzer; Figure 1A), which suggested optimal basal eNOS inhibition by the endogenous Cav-1 expression in EC, which is similar to our in vivo observations (24). In contrast, infection of BAECs with Ad F92A-Cav-1 caused an increase in basal nitrite release (Figure 1A) at both MOI. The expression of endogenous and expressed Cav-1 was confirmed by Western blotting with anti-Cav-1, anti-Myc, or anti-HA antibodies.

In order to determine the mechanism behind the increase in $\mathrm{NO}$ release induced by F92A-Cav-1 in ECs, we used a reconstituted system approach in human embryonic kidney 293 (HEK293) cells that lack all NOS isoforms. Transfection of human eNOS (heNOS) cDNA into HEK cells increased basal NO release, confirming eNOS-derived NO synthesis (Figure 1B). Low levels of endogenous Cav-1 were detected in HEK cells, and cotransfection with increasing amounts 
A

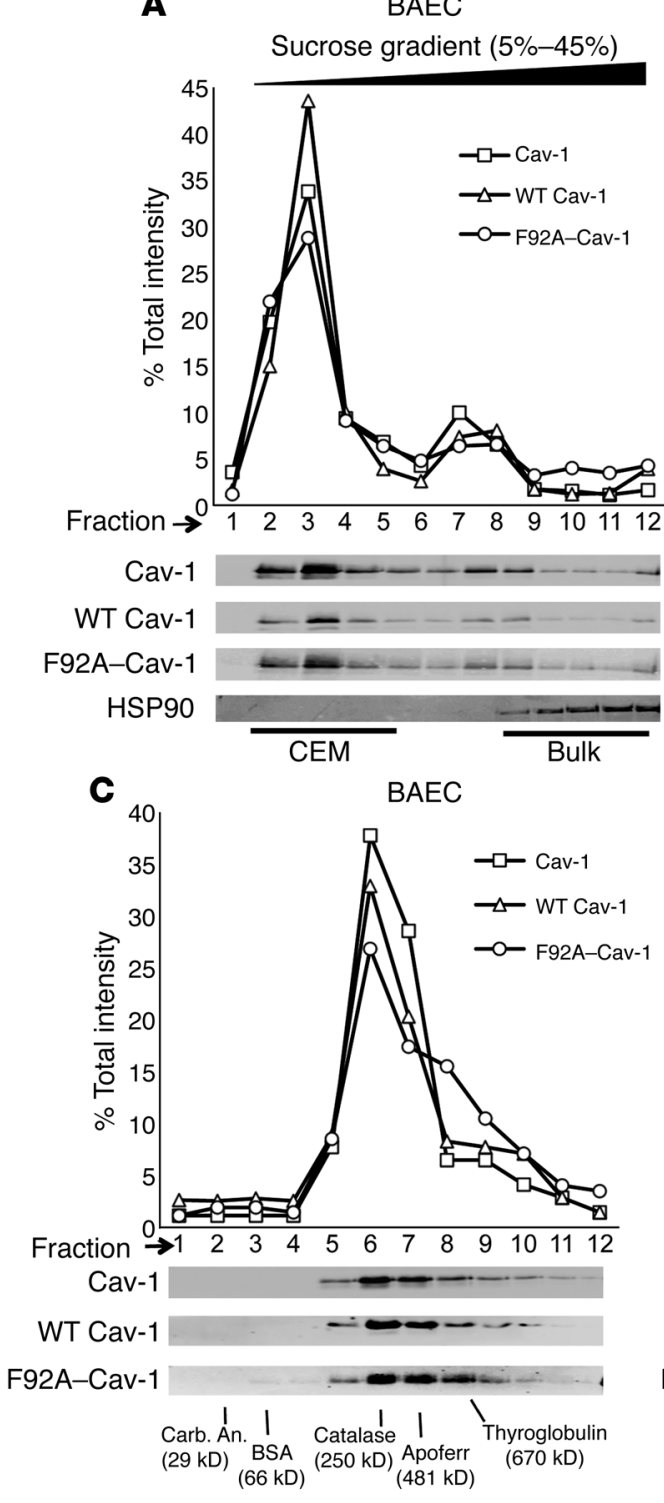

B
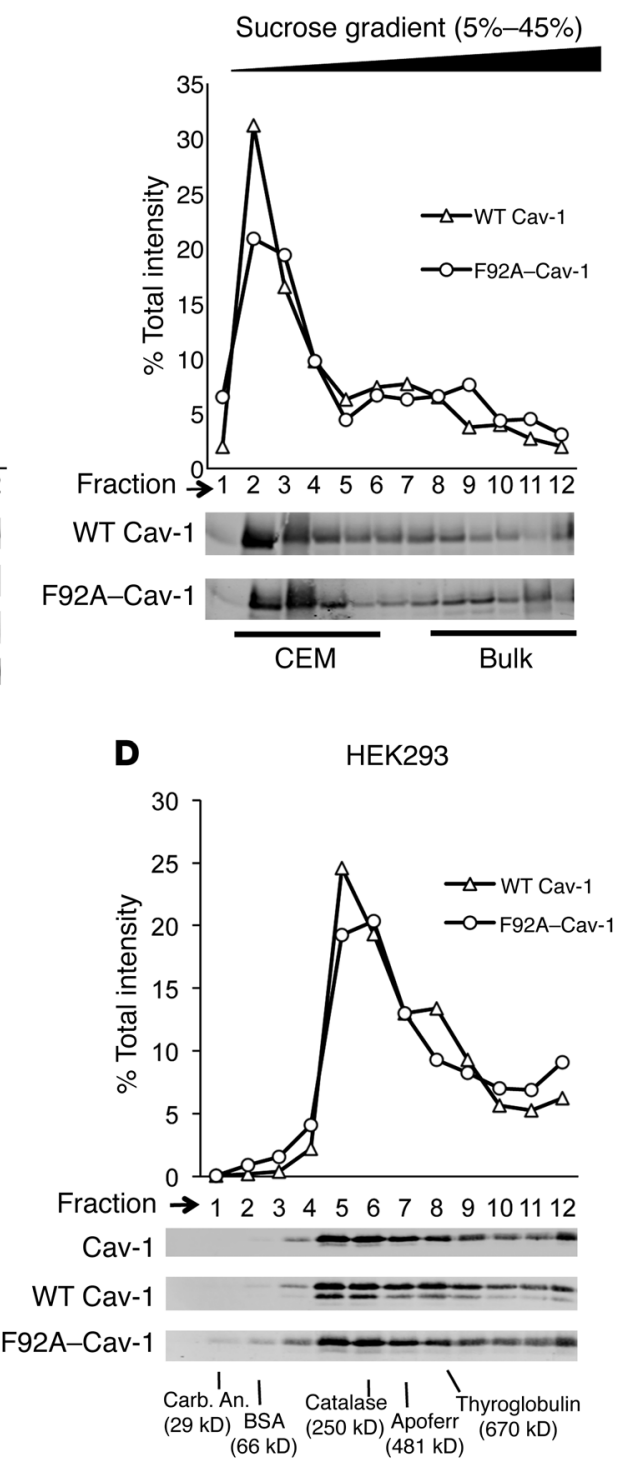

\section{Figure 2}

F92A and WT Cav-1 show similar biochemical properties. (A) BAECs were infected with adenoviruses (25 MOI) encoding for WT (myc tagged) and F92A-Cav-1 (HA tagged) for 48 hours and lysates subjected to sucrose gradient fractionation. Top panel depicts relative distribution of proteins across the gradient (quantified by densitometry), and bottom panels show levels of endogenous Cav-1 (top), WT Cav-1 (myc), F92A-Cav-1 (HA), and HSP90 as a control Immunoblot analysis from fractions collected. Fractions 2 to 5 represent buoyant, CEM fractions, and fractions 8-12 bulk proteins with HSP90 as a marker. (B) HEK293 cells were transfected with WT and F92A-Cav-1 cDNAs and lysates subjected to sucrose fractionation and Western blotting as in A. (C) BAECs were infected as described in A, lysed, and subjected to velocity gradient centrifugation to compare the relative MW of Cav-1 container oligomers. (quantified by densitometry above). Gradients were calibrated with known MW standards. Fractions were blotted for endogenous Cav-1 (top), WT Cav-1 (middle), and F92A-Cav-1 (bottom). The peak level of Cav-1 was in fraction 6 consistent with greater than 10 monomers of Cav-1 (each monomers in $22 \mathrm{kDa}$ ). (D) Expression of WT and F92A-Cav-1 cDNAs in HEK293 cells that express low levels of endogenous Cav-1. Samples were processed as described in C. Similar results were obtained in 2 additional experiments. of the Cav-1 cDNA reduced NO release in a dose-dependent manner as expected in this cell line with lower endogenous Cav-1 levels than ECs (Figure 1B). In contrast, cotransfection of eNOS with increasing amounts of F92A-Cav-1 caused a dose-dependent increase in basal NO release above eNOS transfected alone (Figure 1B). Western blot analysis indicated that eNOS levels were unchanged by WT or F92A-Cav-1 cotransfection, and quantification revealed that the effects of WT and F92A-Cav-1 on NO release were protein expression dependent but diametrically opposed (Figure 1B).

F92A mutation does not modulate the biochemical properties of Cav-1. To better understand how F92A-Cav-1 increases NO release, we determined whether the F92A substitution affects several biochemical properties of Cav-1 by performing sucrose gradient fractionation to isolate Cav-1-enriched microdomains (CEM) followed by independent analysis of Cav-1 oligomerization using velocity sedimentation analysis of molecular complexes. Infection of BAECs with Ad WT Cav-1 and Ad F92A-Cav-1 revealed that about 72\% (myc tagged) and 66\% (HA tagged) of these Cav-1 constructs sedimented in CEM fractions (fractions 2-5) following the pattern of endogenous Cav-1 (Figure 2A). Since it is possible that endogenous Cav-1 may rescue a potential defect caused by F92A substitution, we repeated the above experiments in HEK293 cells expressing lower endogenous Cav-1 levels and similar results were obtained (Figure 2B). Using velocity sedimentation centrifugation, which separates proteins according to their native molecular weights and shapes, WT and F92A-Cav-1 cosedimented with endogenous Cav-1 (Figure 2C), corresponding with approximately 10 monomers of Cav- 1 in the oligomeric complex. Identical results were obtained in the reconstituted system (Figure 2D). These data imply that the F92A substitution does not interfere with 2 of the basic biochemical properties of Cav-1, localization and oligomerization.

F92A-Cav-1 colocalizes and interacts with eNOS similarly to WT Cav-1, but releases eNOS from the inbibitory clamp of endogenous Cav-1. Although the F92A substitution impairs the ability of Cav-1 to inhibit eNOS (23), we tested to determine whether this residue is important for Cav-1 binding to eNOS by performing glutathione-S-transferase 
A

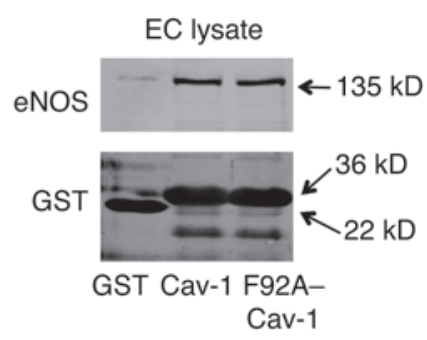

B

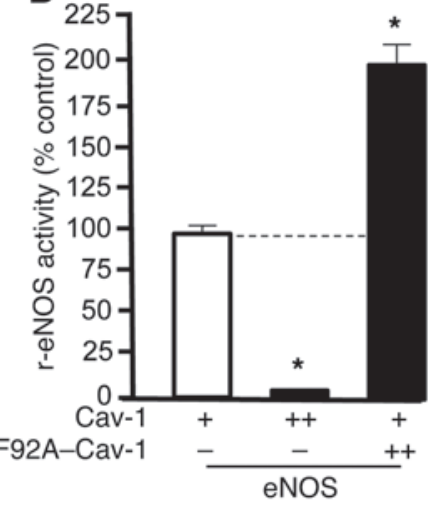

E
C

Recombinant eNOS complex

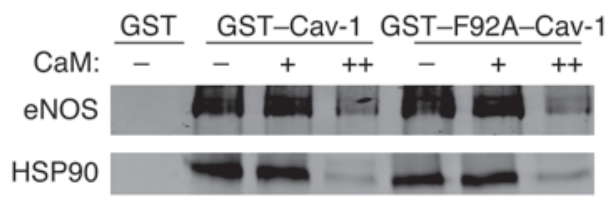

D
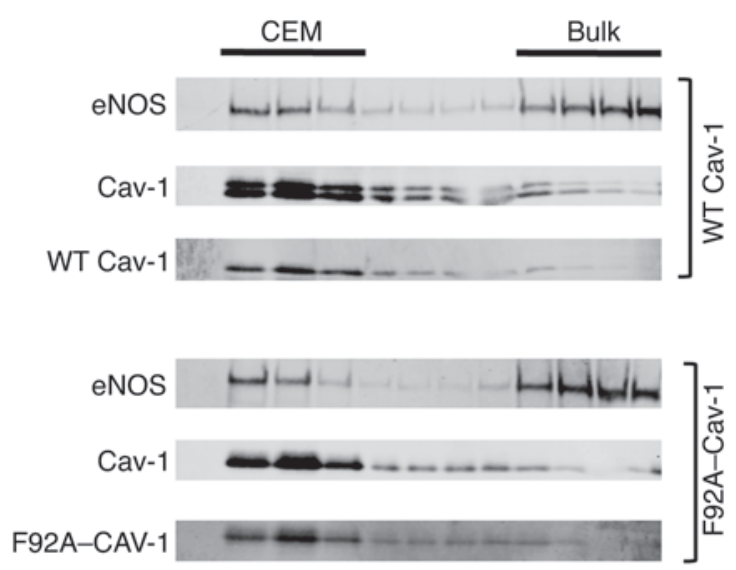

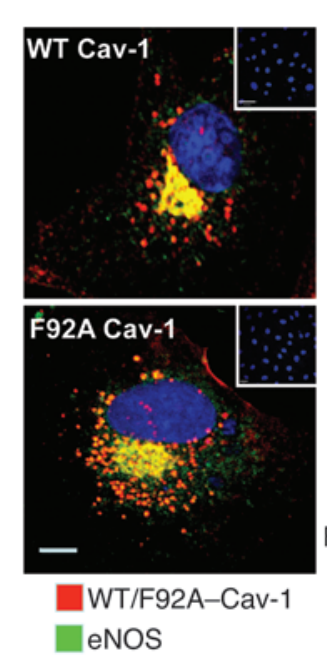

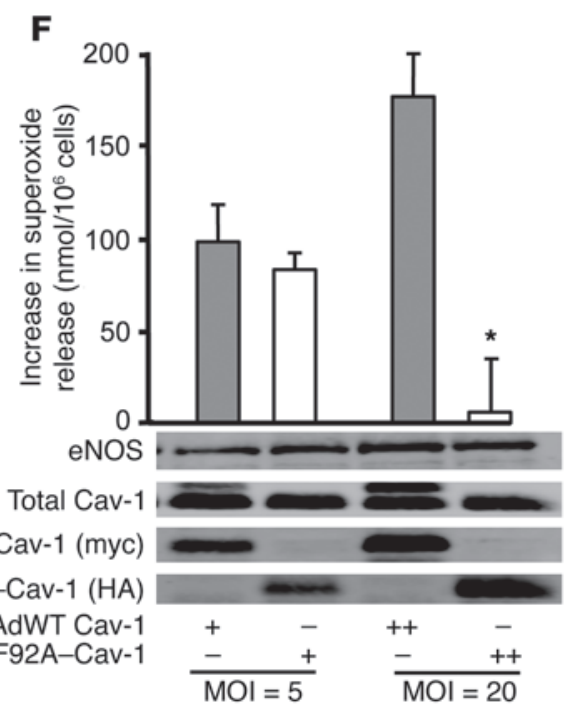

$\mathbf{F}$

Figure 3

F92A-Cav-1 interacts with eNOS and prevents Cav-1 from inhibiting eNOS. (A) BAEC lysates were incubated with GSH-coupled beads coated with GST alone, GST-Cav-1 (aa 62-101), or GST-F92A-Cav-1 (aa 62-101), washed, and eNOS binding determined by immunoblotting. Top panels show interaction of eNOS with Cav-1 domain and the bottom panel documents a similar amount of GST protein input. Performed in triplicate. (B) Recombinant eNOS activity assay. Purified eNOS was incubated with limiting amounts of GST-Cav-1-coated beads (+) or supplemented with excess soluble GST-Cav-1 (++) or excess soluble GST-F92A-Cav-1 (++) and NOS activity quantified. $n=5$ in triplicate. ${ }^{*} P<0.05$. (C) CaM/HSP90-dependent displacement of eNOS binding to GST-Cav-1 and GST-F92A-Cav-1. eNOS $(2 \mu \mathrm{g})$ and HSP90 $(4 \mu \mathrm{g})$ were incubated with beads in the absence $(-)$ or presence of $0.01(+)$ or $1(++) \mu \mathrm{M}$ of CaM. Experiments were performed in triplicate; typical data shown. (D) F92A-Cav-1 does not affect its cosedimentation or trafficking (E) with eNOS. WT Cav-1 (myc, top) or F92A-Cav-1 (HA, bottom) localization was examined by confocal microscopy by using anti-myc/HA (red) or anti-eNOS (green). Nuclei were visualized with DAPI (blue). Inset depicts lack of myc or HA staining in noninfected cells. $n=5$ individual cells. Scale bar: $7 \mu \mathrm{m}$. (F) Decreased superoxide release in BAECs infected with F92A-Cav-1. BAECs were infected with Ad-WT and Ad-F92A-Cav-1 for 48 hours, and superoxide formation was evaluated by cytochrome $C$ reduction. Western blots show protein expression for each condition. $\left(n=7-10 ;{ }^{*} P<0.05\right)$.

(GST) pull-down experiments $(4,25)$. Purified GST, GST-Cav-1 (aa 61-101), and the mutant GST-F92A-Cav-1 (aa 61-101) proteins were expressed and purified from E. coli and incubated with solubilized eNOS prepared from EC lysates. Solubilized eNOS interacted with GST-Cav-1 and GST-F92A-Cav-1 similarly but not with GST alone (Figure 3A). Similar data were obtained using nonacylated, recombinant eNOS purified from E. coli, (Supplemental Figure 1A; supplemental material available online with this article; doi:10.1172/ JCI44778DS1). Therefore, this suggests that the increase in NO release observed with F92A-Cav-1 in eNOS-expressing cells is due to its ability to bind eNOS and "antagonize" the inhibitory clamp of endogenous Cav-1 toward eNOS. To test this idea biochemically, recombinant eNOS activity assays were performed. Under conditions of limited eNOS inhibition by immobilized GST-Cav-1 (Figure 3B; $100 \%$ activity), quantification of L-arginine conversion to the byproduct L-citrulline as a direct assay of eNOS revealed that further addition of soluble GST-Cav-1 markedly reduced eNOS activity as expected, whereas addition of soluble GST-F92A-Cav-1 increased eNOS activity compared with the control conditions (Figure 3B). To examine whether the F92A substitution affected the association of eNOS with other activators of the pathway such as CaM and Hsp90, we compared the binding of eNOS to GST-Cav-1 versus GST-F92ACav-1 using a reconstituted system as previously described (26). The interaction of eNOS and Hsp90 with GST-Cav-1 versus GST-F92ACav-1 was assessed with increasing concentrations of calcium-activated $\mathrm{CaM}(0,0.01,1 \mu \mathrm{M})$. As shown in Figure 3C, the eNOS-Hsp90 
A
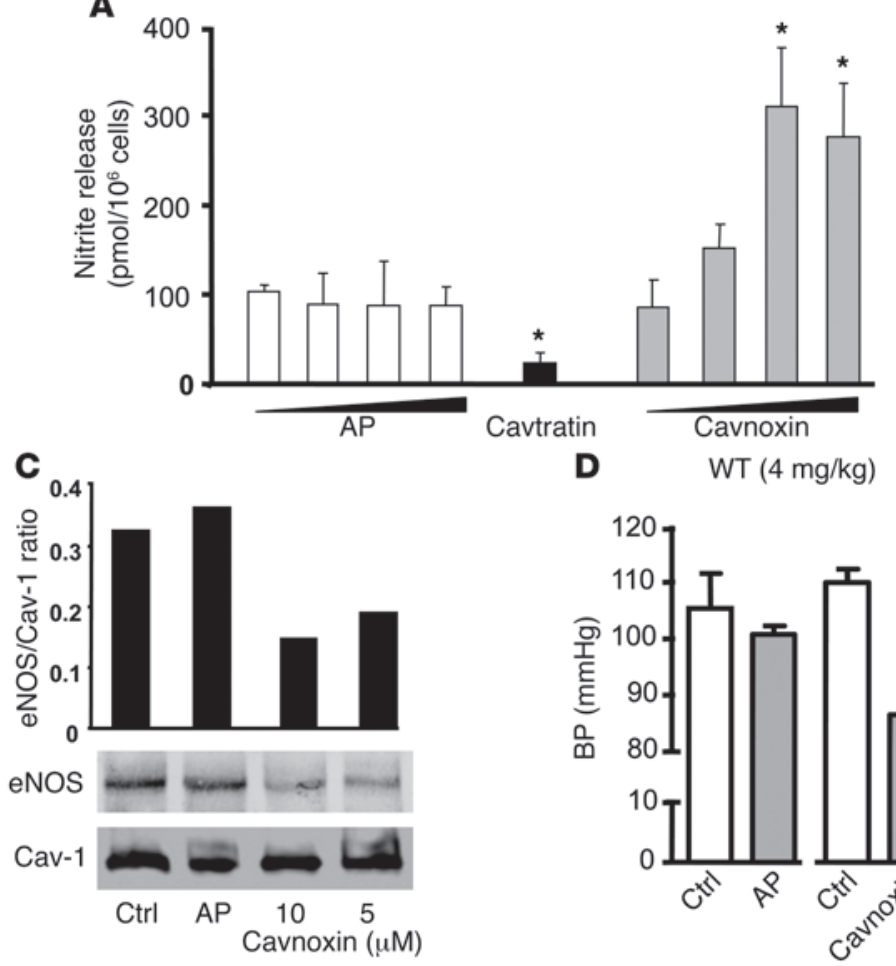

D
B

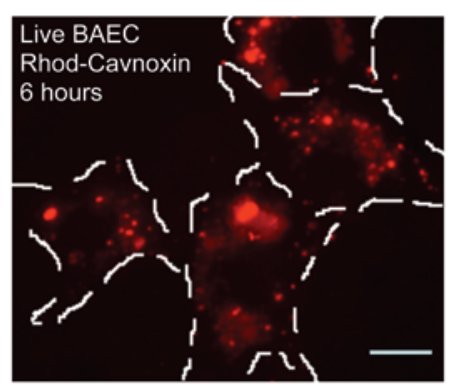

Cavnoxin

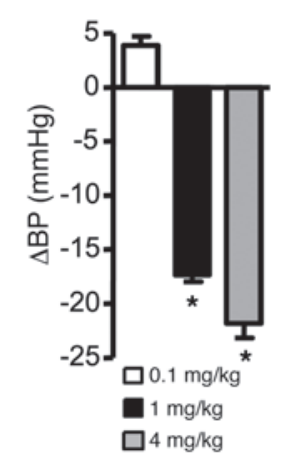

E eNOS KO $(4 \mathrm{mg} / \mathrm{kg})$

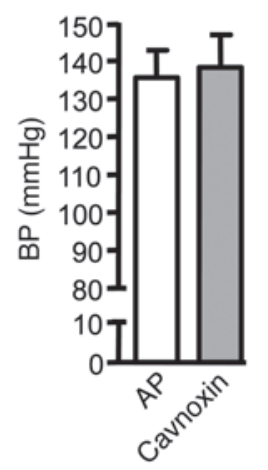

Figure 4

Cavoxin increases NO release and decreases BP in an eNOS-specific fashion. (A) Incubation of ECs with increasing concentrations of Cavnoxin increases basal NO release (12 hours) compared with control peptide (AP), whereas incubation with Cavtratin inhibits NO release. Data are from mean + SEM from $n=4$ in 2 experiments. ${ }^{*} P<0.05$ compared with AP. (B) Uptake of rhodamine-labeled Cavnoxin in unfixed EC. Cells were incubated for 6 hours with peptide $(10 \mu \mathrm{M})$, washed, and visualized by epifluorescence microscopy. Typical data are shown. Scale bar: $20 \mu \mathrm{m}$. (C) Cavnoxin dose dependently decreases eNOS co-IP after Cav-1 IP in BAECs. Cells were left untreated (Ctrl) or treated for 6 hours with AP or Cavnoxin $(5-10 \mu \mathrm{M})$. Cells were lysed and Cav-1 IP performed, followed by blotting for eNOS and Cav-1. Experiments were performed in triplicate. (D) Cavnoxin (4 mg/kg, IP) but not AP (4 mg/kg) decreases mean BP in trained, conscious mice. Right panel demonstrates the effects of Cavnoxin were dose dependent. Data reflect $n=4$ mice per group; ${ }^{\star} P<0.05$ compared with preinjection. (E) Cavnoxin does not lower BP in eNOS KO mice. Experiments were similar to those in $\mathbf{D}$. Data are mean $\pm \mathrm{SEM} ; n=6$ mice.

complex interacts with both GST-Cav-1 and GST-F92A-Cav-1 and also is displaced to the same extent by CaM. Together, these data obtained using purified proteins confirmed that F92A-Cav-1 can increase eNOS activity in the presence of inhibitory WT Cav-1 and can be regulated by similar mechanisms of eNOS activation.

To further confirm that F92A-Cav-1 does not disrupt the other properties of eNOS, we isolated CEM to determine eNOS subcellular localization as well as compare eNOS colocalization with WT and F92A-Cav-1. eNOS showed similar enrichment in both CEM and heavy membrane fractions of both WT and F92A-Cav-1expressing cells, which is typical of this lipid-modified enzyme that targets to Golgi and caveolae membranes (Figure 3D). Localization of eNOS with WT or F92A-Cav-1 in ECs via immunofluorescence microscopy showed that eNOS and expressed WT (Figure 3E) and F92A-Cav-1 (Figure 3E) have similar perinuclear patterns (see individual micrographs in Supplemental Figure 1B) and quantification of the imaging revealed no differences in the colocalization between WT or F92A-Cav-1 with eNOS (Supplemental Figure 1C), signifying that the F92A substitution does not mislocalize Cav-1 or endogenous eNOS.

Under coupled conditions, eNOS largely produces NO, but in the setting of altered redox, eNOS can produce superoxide anion $\left(\mathrm{O}_{2}^{-}\right)$. Thus, we determine whether F92A-Cav-1 influences the pro- duction of $\mathrm{O}_{2}{ }^{-}$in EC using cytochrome $c$ reduction as an assay. Confirmation of $\mathrm{O}_{2}{ }^{-}$release was assessed by preincubating cells with 4-hydroxy-2-nonenal (14), which increased $\mathrm{O}_{2}{ }^{-}$several-fold (not shown). Infection of BAECs with WT or F92A-Cav-1-encoding adenoviruses revealed that at lower MOI, there was no difference in basal $\mathrm{O}_{2}{ }^{-}$produced, but at higher MOI levels, F92A-Cav-1 expression reduced $\mathrm{O}_{2}{ }^{-}$detection (Figure $3 \mathrm{~F}$ ). This suggests that the increased $\mathrm{NO}$ release in F92A-Cav-1-overexpressing cells quenches the basal production of $\mathrm{O}_{2}^{-}$or that F92A-Cav-1 suppresses a source of oxidant generation.

Cavnoxin increases basal NO release from ECs by decreasing eNOS/ Cav-1 interaction. Previously, we have shown that mutation of T90, T91, and in particular, F92 to alanine in Cavtratin (an antennapedia-linked [AP-linked] peptide containing the Cav-1 scaffolding domain, aa 82-101) resulted in the loss of its eNOS inhibitory activity in vitro and in vivo following agonist stimulation (23). Hence, we tested the possibility that an AP-fused mutant scaffolding domain peptide containing the T90/91 and F92A substitutions (called Cavnoxin) could increase basal NO release in vitro. Incubation of cultured EC with AP for 6 hours $(0.1-20 \mu \mathrm{M})$ did not influence NO release, while pretreatment with Cavtratin $(10 \mu \mathrm{M})$ reduced NO release. Under these conditions, Cavnoxin caused a dose-dependent (up to 1.9-fold) increase in NO release compared 

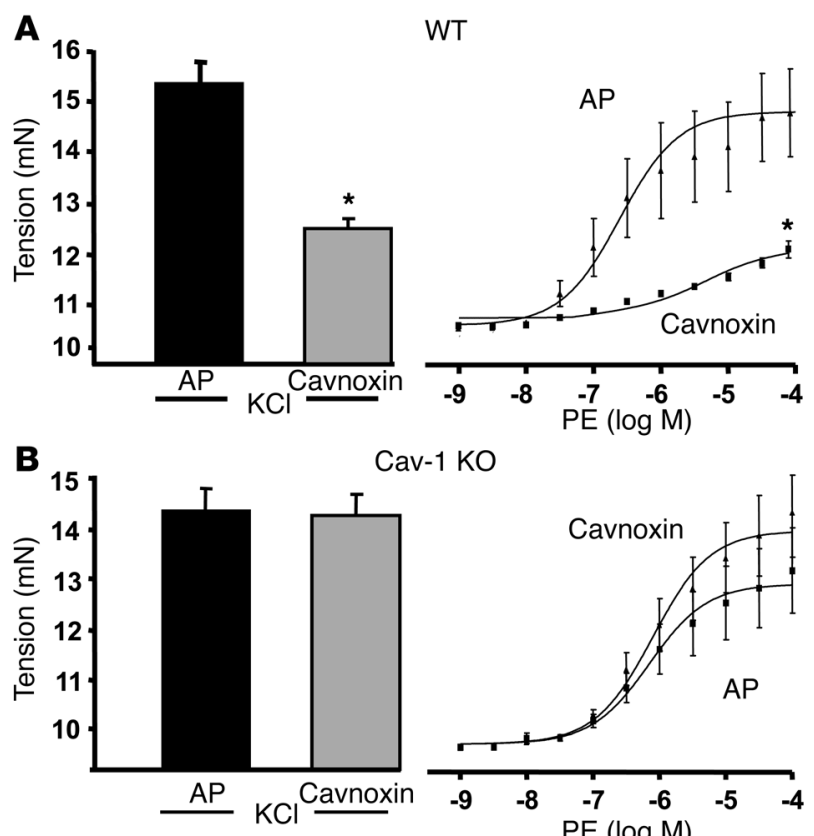

Cav-1 KO
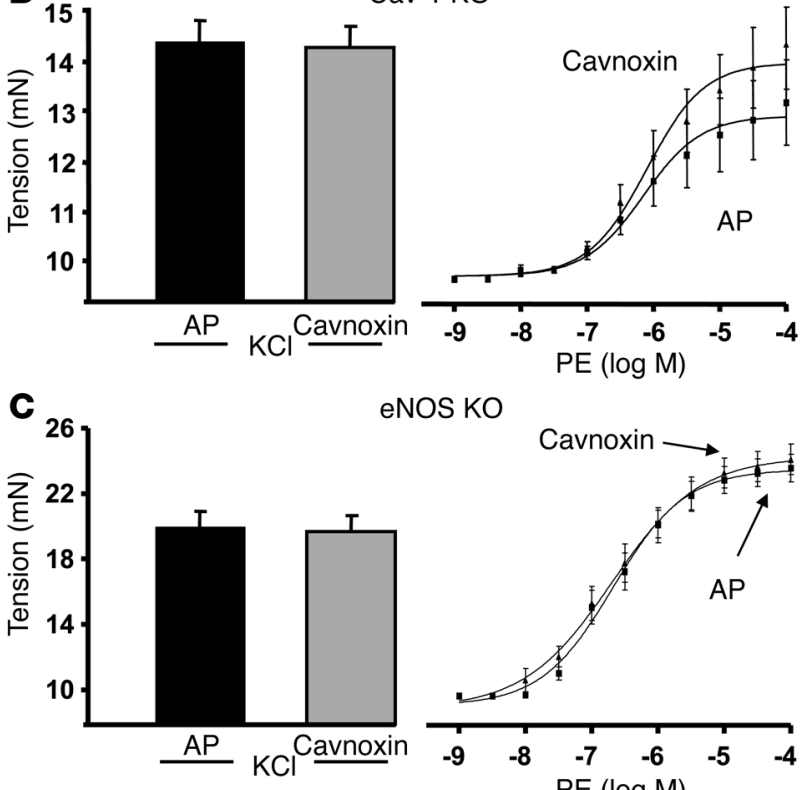

eNOS KO

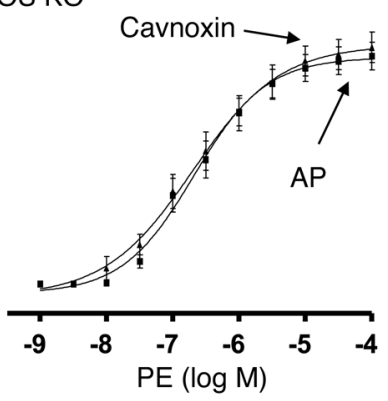

with vehicle-treated ECs (Figure 4A). Pretreatment of live (unfixed) cultured ECs with rhodamine-labeled Cavnoxin confirmed abundant time-dependent cellular uptake (Figure 4B; 6 hour shown) by live cell fluorescence microscopy. No uptake was visible before 15 minutes of incubation, indicating time-dependent internalization of Cavnoxin (data not shown).

To confirm that the increase in NO release caused by Cavnoxin is due to regulated eNOS/Cav-1 interaction, co-IP experiments were performed as previously described for other AP-Cav-derived peptides (23). Pretreatment of EC with Cavnoxin (5-10 $\mu \mathrm{M})$ decreased eNOS association with endogenous Cav-1 compared with control and AP-treated ECs as determined by immunoprecipitating Cav-1 and immunoblotting against eNOS (Figure 4C and quantified as the eNOS/Cav-1 ratio). These data support the idea that Cavnoxin can inhibit the interaction between endogenous Cav-1 and eNOS.

Cavnoxin decreases both BP and vascular tone in an eNOS-dependent manner. Since Cavtratin blocks acute inflammation, tumor growth, and other inflammatory diseases in vivo partially via blockade of NO release $(20,23,26)$, we sought to test whether Cavnoxin could influence eNOS function in vivo. Thus, nonanesthetized C57BL/6 mice were administered AP (as a control) and Cavnoxin (each at 0.1, 1 , and $4 \mathrm{mg} / \mathrm{kg}$ ) and BP measured. AP did not influence BP parameters (Figure 4D), whereas Cavnoxin reduced BP with a threshold effect between 0.1 and $1 \mathrm{mg} / \mathrm{kg}$ (Figure 4D). To assess the specificity

\section{Figure 5}

Cavoxin regulates vascular tone in an eNOS- and Cav-1-dependent manner. (A) Cavnoxin reduces vascular tone in WT vessels. Aortic rings from C57BL/6 mice were incubated with Cavnoxin overnight in organ culture and isometric tension responses to $\mathrm{KCl}$ and $\mathrm{PE}$ examined in myographs; $n=20$ rings from 5 mice. Aortae were isolated from Cav-1 (B) or eNOS-KO (C) mice pretreated with Cavoxin or AP as above. Both $\mathrm{KCl}$ - and $\mathrm{PE}$-induced tension changes were assessed as above. Data are mean \pm SEM, with 4 rings per mouse and 4-5 mice per group.

of Cavnoxin's effect on BP and whether activation of eNOS is necessary for the BP-lowering effect of Cavnoxin, AP and Cavnoxin were administered to eNOS KO mice. As seen in Figure 4E, eNOS KO mice are predictably hypertensive, and administration of Cavnoxin did not lower BP, demonstrating that eNOS is necessary for the BP-lowering effect of the peptide. To confirm the vascular specificity of Cavnoxin's effect, vessels from mice were isolated, incubated overnight with peptides $(10 \mu \mathrm{M})$, and vascular function directly assessed in myographs. Cavnoxin, but not AP, reduced KCl-mediated contractility of the vessels and the contractile response to the $\alpha$ adrenoceptor agonist, phenylephrine (PE) (Figure 4F). Pretreatment with NOS inhibitor, L-NAME, rescued PE-induced constriction by approximately $70 \%-80 \%$, indicating that the actions of the peptide were mostly NO dependent (not shown).

Genetic evidence demonstrates that Cavnoxin requires both eNOS and endogenous Cav-1 to influence vascular tone. With the assumption that Cavnoxin's effect on EC NO release, BP, and vascular tone are mediated through decreased eNOS inhibition by endogenous Cav-1, its effect should require the presence of both Cav-1 and eNOS. Aortic rings isolated from WT or Cav-1 KO mice were incubated with $\mathrm{AP}$ or Cavnoxin as before and vascular responses to $\mathrm{KCl}$ and PE examined. As seen in Figure 5A, Cavnoxin reduced agonistinduced tension development in WT vessels but did not influence $\mathrm{KCl}-$ or PE-induced tone in vessels isolated from Cav-1 $\mathrm{KO}$ mice (Figure 5B), further confirming that Cavnoxin's effect on vascular tone is through regulated eNOS/Cav-1 antagonism. However, Cav-1 is expressed in both ECs and SMCs. To confirm the eNOS and vascular endothelial specificity of Cavnoxin, similar experiments were performed in aortic rings from eNOS-null mice, in which eNOS is specifically expressed in the endothelial monolayer. As shown in Figure 5C, Cavnoxin had no effect in such vessels. Together, these data show that Cavnoxin's vascular effect is Cav-1and eNOS-dependent and confirm the lack of a direct effect on SMC contractility. Consequently, F92A-Cav-1 and Cavnoxin seem to downregulate eNOS binding and inhibition by endogenous Cav-1, shifting the eNOS balance toward an "uninhibited or less inhibited eNOS" and leading to increased NO release and vasodilation (as summarized in the model in Figure 6).

\section{Discussion}

Our results demonstrate that targeted mutation of the Cav-1 scaffolding domain reduces the inhibitory influence of endogenous Cav-1 toward eNOS and increases eNOS-derived NO. Mutation of F92 in Cav-1 does not influence its biochemical or biophysical properties such as flotation on sucrose gradients, sedimentation velocity and oligomerization, cellular localization, or interaction with eNOS in vitro. However, F92A-Cav-1 increases NO release from cells expressing eNOS. Mechanistic experiments using purified eNOS, GST-Cav-1, and GSTF92A-Cav-1 confirm that this occurs via F92A-Cav-1 binding 

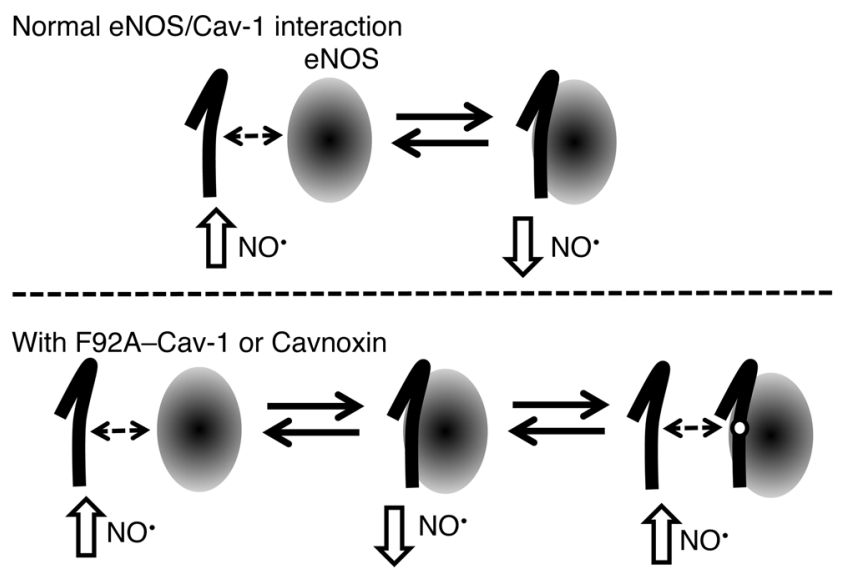

Figure 6

Proposed mechanism of increased eNOS NO release by Cavnoxin and F92A-Cav-1. eNOS (oval) is thought to exist in a dynamic balance between a "more active" state (free of Cav-1 inhibitory clamp, left side) and a "less active" state (bound to and inhibited by endogenous Cav-1, lower panel). Our data show that F92A-Cav-1 and Cavnoxin can bind eNOS (left) and prevent its inhibition by endogenous Cav-1, resulting in the creation of a third dynamic state characterized by an eNOS bound to a noninhibitory Cav-1 scaffolding domain containing the F92A substitution (lower panel, far right), thereby shifting eNOS equilibrium toward a "more active" state by preventing the inhibitory clamp of endogenous Cav-1.

to eNOS and thus removing the inhibitory influence of endogenous Cav-1 on eNOS. Moreover, a cell-permeable peptide harboring a mutant scaffolding domain called Cavnoxin increases NO release, decreases eNOS binding to endogenous Cav-1 from cultured ECs, reduces vascular tone, and lowers BP in an eNOS-dependent manner, since the pressure-lowering actions of Cavnoxin are eliminated in eNOS KO mice. The beneficial actions of Cavnoxin on isolated vessels require both endogenous Cav-1 and eNOS, since the vascular actions of the peptide are eliminated in both $\mathrm{KO}$ strains. Thus, these data document the feasibility of improving vascular function by interfering with the inhibitory influence of endogenous Cav-1 on eNOS and suggest this interaction as a potential pharmacological target.

Mounting biochemical and genetic evidence has shown that eNOS is highly regulated by Cav-1 through a direct protein-protein interaction. However, little is known about the extent by which eNOS is inhibited by Cav-1 under physiological, nonstimulated conditions, since the loss of Cav-1 abrogates the interaction with eNOS as well as the formation of caveolae in EC (i.e., Cav-1 control of eNOS function cannot be dissociated from its role in caveolae assembly). Herein, we show that the F92A-Cav-1 can interact with eNOS and promote NO release. Since the biochemical assays performed indicate that F92A-Cav-1 behaves identically to WT Cav-1, our data are the first, to our knowledge, to specifically demonstrate an increase in eNOS-derived NO release as a result of decreased Cav-1 inhibitory clamp without interfering with other functions of endogenous Cav-1 (to form caveolae). Moreover, this mutant may be useful to separate the functions of Cav-1 in regulating other signaling pathways that rely on the scaffolding domain function of Cav-1 from its structural role in organelle assembly. However, in vivo experiments with Cavnoxin in animals or vessels lacking eNOS or Cav-1 are consistent with eNOS as the critical target regulating vascular function and BP, and reveal that Cavnoxin has a high degree of specificity toward the eNOS/Cav-1 interaction.

In summary, here we show that targeted interference with the inhibitory actions of endogenous Cav-1 promotes NO release, augments vessel function, and lowers BP. Since eNOS-derived NO is critical for normal cardiovascular homeostasis, our results suggest that it is feasible to modulate eNOS function via perturbation of its protein-protein interaction with Cav-1. Although activation of eNOS is thought to be generally beneficial for the cardiovascular system, under certain circumstances, in particular in mice lacking Cav-1, sustained uncoupled eNOS may promote to pulmonary hypertension and cardiac hypertrophy (11). We favor the idea that Cavnoxin is unique in its action in regulating the beneficial aspects of eNOS activation, since caveolae are still present and functional in normal mice, as opposed to Cav-1 KO mice, where there are several pathways that are dysregulated, including lipid metabolism, calcium homeostasis, and mechanotransduction. Clearly, future studies aimed at optimization of Cavnoxin and potential small molecule design based on Cavnoxin would afford novel opportunities to improve endothelial function.

\section{Methods}

Cell culture. BAECs below passage 15, COS cells, and HEK cells were cultured in DMEM (Invitrogen) supplemented with 5\% FBS and $1 \times$ penicillin/streptomycin in a humidified incubator at $37^{\circ} \mathrm{C}$ with $7 \% \mathrm{CO}_{2}$ as described.

Cell transfection and infection. Cell transfection was carried out as previously described (23). Plasmid amounts were normalized to ensure equal DNA quantities. Cell proteins were isolated 48 hours later for experiments with the appropriate buffer. For cell infection, semiconfluent BAECs grown in a 100-mm tissue culture dish were infected with adenoviruses encoding for WT Cav-1 (myc-tag) and F92A-Cav-1 (HA-tag). Cell proteins were isolated 48 hours later for experiments. Peptides were designed and synthesized as previously described (23). Cavnoxin refers to mutant CSD peptide (DGIWKASFAAATVTKWYFYR) fused to AP (RQIKIWFQNRRMKWKK).

Isolation of caveolae/lipid rafts, NO accumulation, and eNOS activity. Isolation of Caveolae/lipid rafts was performed by a detergent-free fractionation method as previously described $(1,23)$. Treatment of cells and quantification of NO release (measured as nitrite) were performed as previously described (23) with a NO-specific chemiluminescence analyzer (NOAi; GE). NOS-specific NO quantification was confirmed by ensuring that more than $95 \%$ of responses were blocked by NOS inhibitor L-NAME. eNOS activity assays were performed as previously described (26).

Velocity gradient centrifugation. Cells (2 confluent 100-mm dishes) were lysed with $500 \mu \mathrm{l}$ of Mes-buffered saline (MBS; $25 \mathrm{mM} \mathrm{Mes,} \mathrm{pH} \mathrm{6.5/0.15} \mathrm{M}$ $\mathrm{NaCl}) / 60 \mathrm{mM} \beta$-octyl glucoside. Cellular aggregates were dissociated by sonication (three 10-second bursts), and the solubilized material was then loaded on top of a $5 \%-45 \%$ continuous sucrose gradient prepared in MBS/60 $\mathrm{mM} \beta$-octyl glucoside $(12 \mathrm{ml})$ and centrifuged at $80,000 \mathrm{~g}$ for 16-20 hours. Following centrifugation, twelve 1-ml fractions were collected from the gradient and Western blot analysis performed. To better estimate the molecular mass of the proteins from the gradient, velocity gradient centrifugation was carried out on 5 molecular weight standards: thyroglobulin $(670 \mathrm{kDa})$, apoferritin $(480 \mathrm{kDa})$, catalase $(250 \mathrm{kDa})$, BSA $(66 \mathrm{kDa})$, and carbonic anhydrase $(29 \mathrm{kDa})$.

Purification of GST-Cav-1 fusion proteins (WT and F92A-Cav-1) and in vitro interactions. Caveolin fusion protein expression was performed as described previously $(4,25,26)$. To determine binding of GST-Cav- 1 and GST-F92A-Cav-1 to eNOS, a GST pull-down assay using BAEC lysates was performed. Cells were lysed in a STET buffer (50 mM Tris-Cl, pH 7.4, 
$150 \mathrm{mM} \mathrm{NaCl}, 1 \mathrm{mM}$ EDTA, 1\% Triton X-100) containing 1× protease inhibitors and $1 \mathrm{mM}$ PMSF. The samples were Dounce homogenized (20 strokes) and tumbled for 1 hour at $4^{\circ} \mathrm{C}$. Following this, samples were centrifuged for 10 minutes at $5,000 \mathrm{~g}$ at $4^{\circ} \mathrm{C}$, sonicated (three 10 -second bursts), and incubated overnight at $4^{\circ} \mathrm{C}$ with beads containing GST alone, GST-Cav-1, or GST-F92A-Cav-1 ( $80 \mu \mathrm{l}$ packed volume) in a total volume of $500 \mu \mathrm{l}$. After binding, the beads were washed (three 5 -minute intervals) with wash buffer containing $50 \mathrm{mM}$ Tris- $\mathrm{Cl}$ ( $\mathrm{pH} 7.4), 125 \mathrm{mM}$ $\mathrm{NaCl}, 1 \mathrm{mM}$ EDTA, and $1 \mathrm{mM}$ EGTA. Furthermore, in vitro interactions of GST-Cav-1 and GST-F92A-Cav-1 with recombinant eNOS (Cayman Chemicals) were performed, as previously described (26) for 2 hours at $4{ }^{\circ} \mathrm{C}$ in binding buffer $(50 \mathrm{mM}$ Tris- $\mathrm{Cl}, \mathrm{pH} 7.4$, in $20 \%$ glycerol). After binding, beads were washed (five 5 -minute intervals) with a high salt wash buffer (50 mM Tris-Cl, pH 7.7, $400 \mathrm{mM} \mathrm{NaCl}$, and $1 \mathrm{mM}$ EDTA). Beads were eluted by boiling in $1 \times$ SDS sample buffer and subjected to SDS/PAGE gel electrophoresis followed by Western blotting with antiGST and anti-eNOS antibodies. Specificity of interaction with the Cav-1 scaffolding domain (aa 82-101) was confirmed by the lack of interaction of eNOS to GST alone, GST-Cav-1 (aa 61-81), or GST-Cav-1 (aa 61-81) fused to a 20 aa polyalanine sequence.

Immunofluorescence. Immunofluorescence experiments were performed on BAECs infected with WT and F92A-Cav-1 adenoviruses. BAECs were seeded in gelatin-coated coverslips and infected as described above. Fortyeight hours after infection start, immunofluorescence staining was performed as previously described (23) and visualized by confocal microscopy. For uptake studies, BAECs were incubated with rhodamine-labeled Cavnoxin for up to 6 hours at $10 \mu \mathrm{M}$, followed by washing and visualization. No peptide aggregation was observed.

Vascular reactivity assays. Animal experiments were approved by the IACUC at the Yale University School of Medicine. WT and eNOS-null mice were purchased from Charles River or JAX, respectively, whereas Cav-1 KO mice were bred in-house as previously described (18). Long-term husbandry was done at Yale University; mice were fed normal chow diet. Isolation and pretreatment of aortic rings as well as vascular reactivity assays were performed as previously described $(20,24)$.

Quantification of superoxide $\left(\mathrm{O}_{2}^{-}\right)$release. Cytochrome $c$ reduction assays were adapted from previous work (27). BAECs were seeded in 24-well plates and infected with adenoviruses. Forty-eight hours after infection, cells were washed twice with PBS and incubated under low lighting conditions with a PBS-based $(\mathrm{Ca} 2+/ \mathrm{Mg} 2+)$ buffer containing $50 \mu \mathrm{M}$ acetylated ferricytochrome $c$ (Sigma-Aldrich) and catalase (125 units/ml; Sigma-Aldrich) for 30 minutes in a $37^{\circ} \mathrm{C}$ incubator. The postincubation supernatant was then removed from each well, transferred into labeled Eppendorf tubes (1 tube/ well), and placed on ice. Immediately, the cells were incubated for an additional 30 minutes in similar conditions with superoxide dismutase (SOD)
(200 units/ml; Sigma-Aldrich). In the meantime, absorbance at $540 \mathrm{~nm}$, $550 \mathrm{~nm}$, and $560 \mathrm{~nm}$ was measured by using a spectrophotometer (Magellan; TECAN) followed by OD measurement of the SOD-containing medium. The amount of $\mathrm{O}_{2}^{-}$produced from each well was calculated using the following conversion equations: $\triangle O D 550 \mathrm{~nm}=$ OD $550 \mathrm{~nm}-([\mathrm{OD} 540 \mathrm{~nm}+$ $\mathrm{OD} 560 \mathrm{~nm}) / 2])$ and $\mathrm{O}_{2}^{-}=(\Delta \mathrm{OD} 550 \mathrm{~nm}$ without $\mathrm{SOD}-\Delta \mathrm{OD} 550 \mathrm{~nm}$ with SOD) $/ 21.1 \mathrm{mmol} / \mathrm{L}-1 \mathrm{~cm}-1$ where $\Delta$ represents change in absorbance at $550 \mathrm{~nm}$ and $21.1 \mathrm{mmol} / \mathrm{L}-1 \mathrm{~cm}-1$ is the extinction coefficient for $\mathrm{O}_{2}{ }^{-}$. Validation of $\mathrm{O}_{2}{ }^{-}$quantification was confirmed by exposing cells to 4-hydroxy2-nonenal or angiotensin II.

$B P$ measurements. BP measurements were performed in nonanesthetized aged mice (1 year old) using the Coda- 6 tail cuff system starting at approximately 10 am each day. Mice were preconditioned over 3-5 days with daily mock measurements in regular chambers. BP was determined at baseline and at 1 hour following i.p. injection of either vehicle peptide or varying concentrations of Cavnoxin peptide (18). BP for each mouse was taken to be the average of data collected over 10 consecutive cuff inflation cycles following 20-25 conditioning cycles.

Statistics. Data are presented as mean \pm SEM and considered as statistically significant at $P<0.05$ by ANOVA followed by Dunnet's $t$ test.

\section{Acknowledgments}

This work was supported by grants R01 HL64793, R01 HL61371, R01 HL081190, HL096670, and P01 HL70295 from the NIH to W.C. Sessa, and grants from the Canadian Institutes for Health Research (CIHR), the Michael Smith Foundation for Health Research (MSFHR), the Canadian Foundation for Innovation, and the British Columbia Knowledge Development Fund to P. Bernatchez. A. Sharma and P. Bernatchez are supported by salary awards from CIHR and MSFHR. E.P. Marin was supported by a postdoctoral research fellowship from the National Kidney Foundation and is presently supported by a K08 Award from the NIH.

Received for publication August 16, 2010, and accepted in revised form June 1, 2011.

Address correspondence to: William C. Sessa, Department of Pharmacology and Vascular Biology and Therapeutics Program, Yale University School of Medicine, 10 Amistad St., New Haven, Connecticut 06520, USA. Phone: 203.737.2291; Fax: 203.737.2290; E-mail: william.sessa@yale.edu. Or to: Pascal Bernatchez, Providence Heart and Lung Institute, St. Paul's Hospital, James Hogg Research Centre, 1081 Burrard St., Room 166, Vancouver (BC) Canada, V6Z 1Y6. Phone: 604.682.2344, ext. 66060; Fax: 604.806.9274; E-mail: pbernatc@interchange.ubc.ca.
1. Garcia-Cardena G, Oh P, Liu J, Schnitzer JE, Sessa WC. Targeting of nitric oxide synthase to endothelial cell caveolae via palmitoylation: implications for nitric oxide signaling. Proc Natl Acad Sci US A. 1996;93(13):6448-6453.

2. Sessa WC, et al. The Golgi association of endothelial nitric oxide synthase is necessary for the efficient synthesis of nitric oxide. J Biol Chem. 1995; 270(30):17641-17644.

3. Parton RG, Simons K. The multiple faces of caveolae. Nat Rev Mol Cell Biol. 2007;8(3):185-194.

4. Garcia-Cardena G, et al. Dissecting the interaction between nitric oxide synthase (NOS) and caveolin. Functional significance of the nos caveolin binding domain in vivo. J Biol Chem. 1997; 272(41):25437-25440.

5. Michel JB, Feron O, Sacks D, Michel T. Reciprocal regulation of endothelial nitric-oxide synthase by
Ca2+-calmodulin and caveolin. J Biol Chem. 1997; 272(25):15583-15586.

6. Ju H, Zou R, Venema VJ, Venema RC. Direct interaction of endothelial nitric-oxide synthase and caveolin-1 inhibits synthase activity. J Biol Chem. 1997; 272(30):18522-18525.

7. Drab M, et al. Loss of caveolae, vascular dysfunction, and pulmonary defects in caveolin-1 gene-disrupted mice. Science. 2001;293(5539):2449-2452.

8. RazaniB,etal.Caveolin-1 nullmiceareviablebutshowevidence of hyperproliferative and vascular abnormalities. J Biol Chem. 2001;276(41):38121-38138.

9. Wunderlich C, et al. Chronic NOS inhibition prevents adverse lung remodeling and pulmonary arterial hypertension in caveolin-1 knockout mice. Pulm Pharmacol Ther. 2008;21(3):507-515.

10. Wunderlich $\mathrm{C}$, et al. Nitric oxide synthases are crucially involved in the development of the severe car- diomyopathy of caveolin-1 knockout mice. Biochem Biophys Res Commun. 2008;377(3):769-774.

11. Zhao YY, et al. Persistent eNOS activation secondary to caveolin-1 deficiency induces pulmonary hypertension in mice and humans through PKG nitration. J Clin Invest. 2009;119(7):2009-2018.

12. Lisanti MP, Tang ZL, Sargiacomo M. Caveolin forms a hetero-oligomeric protein complex that interacts with an apical GPI-linked protein: implications for the biogenesis of caveolae. J Cell Biol. 1993;123(3):595-604.

13. Sargiacomo $M$, et al. Oligomeric structure of caveolin: implications for caveolae membrane organization. Proc Natl Acad Sci US A. 1995;92(20):9407-9411.

14. Whitsett J, Picklo MJ Sr, Vasquez-Vivar J. 4-Hydroxy-2-nonenal increases superoxide anion radical in endothelial cells via stimulated GTP cyclohydrolase proteasomal degradation. Arterio- 
scler Thromb Vasc Biol. 2007;27(11):2340-2347.

15. Fulton D, Gratton JP, Sessa WC. Post-translational control of endothelial nitric oxide synthase: why isn't calcium/calmodulin enough? J Pharmacol Exp Ther. 2001;299(3):818-824.

16. Michel JB, Feron O, Sase K, Prabhakar P, Michel T. Caveolin versus calmodulin. Counterbalancing allosteric modulators of endothelial nitric oxide synthase. J Biol Chem. 1997;272(41):25907-25912.

17. Sessa WC. eNOS at a glance. J Cell Sci. 2004; 117(pt 12):2427-2429.

18. Murata T, et al. Reexpression of caveolin- 1 in endothelium rescues the vascular, cardiac, and pulmonary defects in global caveolin-1 knockout mice. J Exp Med. 2007;204(10):2373-2382.

19. Yu J, et al. Direct evidence for the role of caveolin-1 and caveolae in mechanotransduction and remodeling of blood vessels. J Clin Invest. 2006;
116(5):1284-1291.

20. Bucci M, et al. In vivo delivery of the caveolin-1 scaffolding domain inhibits nitric oxide synthesis and reduces inflammation. Nat Med. 2000;6(12):1362-1367.

21. Chidlow JH Jr, et al. Endothelial caveolin-1 regulates pathologic angiogenesis in a mouse model of colitis. Gastroenterology. 2009;136(2):575-584.

22. Gratton JP, et al. Selective inhibition of tumor microvascular permeability by cavtratin blocks tumor progression in mice. Cancer Cell. 2003; 4(1):31-39.

23. Bernatchez PN, Bauer PM, Yu J, Prendergast JS, He P, Sessa WC. Dissecting the molecular control of endothelial NO synthase by caveolin-1 using cellpermeable peptides. Proc Natl Acad Sci U S A. 2005; 102(3):761-766.

24. Bauer PM, et al. Endothelial-specific expression of caveolin-1 impairs microvascular permeability and angiogenesis. Proc Natl Acad Sci U S A. 2005; 102(1):204-209.

25. Couet J, Li S, Okamoto T, Ikezu T, Lisanti MP. Identification of peptide and protein ligands for the caveolin-scaffolding domain. Implications for the interaction of caveolin with caveolae-associated proteins. J Biol Chem. 1997;272(10):6525-6533.

26. Gratton JP, Fontana J, O'Connor DS, GarciaCardena G, McCabe TJ, Sessa WC. Reconstitution of an endothelial nitric-oxide synthase (eNOS), hsp90, and caveolin-1 complex in vitro. Evidence that hsp90 facilitates calmodulin stimulated displacement of eNOS from caveolin-1.J Biol Chem. 2000;275(29):22268-22272.

27. Dikalov S, Griendling KK, Harrison DG. Measurement of reactive oxygen species in cardiovascular studies. Hypertension. 2007;49(4):717-727. 